\title{
42. LEG 23 BIOSTRATIGRAPHIC SYNTHESIS: LOWER EOCENE TO PLEISTOCENE OF THE ARABIAN SEA*
}

\author{
Catherine Nigrini, Lexington, Massachusetts, \\ Robert L. Fleisher, University of Southern California, Los Angeles, California, \\ and \\ Joseph E. Boudreaux, Texaco Inc., New Orleans, Louisiana
}

By integrating the sediment cores recovered at Sites 219 and 220 , a fairly complete Tertiary sequence can be obtained, and a correlation between the sites can be made despite occasional drilling and recovery gaps. Foraminifera and calcareous nannofossils are common and well preserved throughout the sequence; Radiolaria are found in numbers in Upper Oligocene through Lower Eocene sediments. More detailed studies of the fossil groups may be found in other chapters within this volume.

This compilation of paleontological events from Arabian Sea sediments is preliminary and should be refined and expanded as additional core material becomes available. Similar compilations for other parts of the Indian Ocean would provide the basis for the construction and correlation of regional biostratigraphic zonation systems. A synthesis of this type has already been made for the western tropical Pacific (Brönnimann et al., 1971).

In Table 1, paleontological events for the calcareous nannofossils (highest species occurrences), foraminifera (highest and lowest species occurrences), and Radiolaria (highest and lowest species occurrences and evolutionary transitions) have been tabulated chronologically. The top of the range of a taxon is indicated by $\mathrm{T}$, the bottom by $\mathrm{B}$ and an evolutionary transition by an arrow.

The interval within which these events occurred, expressed in terms first of cores and sections and then of subbottom depth (in meters), is given in the body of the
Table. It should be noted that a drilled interval often produces artificial bunching of events.

Table 2 shows the relationship between zonations based on calcareous nannofossils, foraminifera, and Radiolaria, as observed at Sites 219 and 220. The thickness allocated to each zone is proportional to the thicknesses at the two sites. Dashed lines in the foraminiferal column indicate unconformities, and hatchured areas represent drilled intervals. Boundaries between epochs are based on calcareous nannofossil data; this convention has been used in the site summary chapters unless overwhelming evidence from another fossil group has dictated the boundary position. There are discrepancies in the placement of the Pliocene-Miocene boundary and the Late-Middle Miocene boundary in Site 219 (see the biostratigraphic summary for Site 219). The correlations between Sites 219 and 220 differ slightly for each fossil group, producing offsets in the Eocene portion of Table 2.

\section{REFERENCE}

Bronnimann, P. et al., 1971. Biostratigraphic synthesis: Late Oligocene and Neogene of the Western tropical Pacific. In Winterer, E. L., Riedel, W. R., et al., Initial Reports of the Deep Sea Drilling Project, Volume VII: Washington (U.S. Government Printing Office), p. 1723-1745
*"Documentation for radiolarian events in this chapter will appear in an article by Catherine Nigrini to be published in Volume 24 of the Initial Report series." 
TABLE 1

Sequence of Paleontological Events Recognized in the Arabian Sea

(Events are from youngest downward-see text for details)

\begin{tabular}{|c|c|c|c|c|c|}
\hline \multicolumn{2}{|c|}{ Calcareous Nannofossils } & \multicolumn{2}{|l|}{ Foraminifera } & \multicolumn{2}{|l|}{ Radiolaria } \\
\hline Events & $\begin{array}{l}\text { Interval: Site, } \\
\text { Core, Section, } \\
\text { Subbottom } \\
\text { Depth (m) }\end{array}$ & Events & $\begin{array}{l}\text { Interval: Site, } \\
\text { Core, Section, } \\
\text { Subbottom } \\
\text { Depth }(\mathrm{m})\end{array}$ & Events & $\begin{array}{l}\text { Interval: Site, } \\
\text { Core, Section, } \\
\text { Subbottom } \\
\text { Depth (m) }\end{array}$ \\
\hline \multirow[t]{5}{*}{$\mathrm{T}$ Gephyrocapsa oceanica } & $\begin{array}{l}\text { Within } \\
219-1-1 \\
(0-2)\end{array}$ & B Globigerinella adamsi & $\begin{array}{l}219-1-1 \\
219-1-2 \\
(0-2)\end{array}$ & & \\
\hline & & T Streptochilus tokelauae & $\begin{array}{l}219-1-1 \\
219-1-2 \\
(0-2)\end{array}$ & & \\
\hline & & $\begin{array}{l}\text { T Turborotalia (Turborotalia) } \\
\text { pseudopima }\end{array}$ & $\begin{array}{l}219-1-1 \\
219-1-2 \\
(0-2)\end{array}$ & & \\
\hline & & $\begin{array}{l}\mathrm{T} \text { Globorotalia (Globorotalia) } \\
\text { tumida flexuosa }\end{array}$ & $\begin{array}{l}219-1-2 \\
219-1-3 \\
(2-3)\end{array}$ & & \\
\hline & & $\mathrm{T}$ Globigerina digitata & $\begin{array}{l}219-1-3 \\
219-1-4 \\
(3-5)\end{array}$ & & \\
\hline \multirow[t]{4}{*}{ T Pseudoemiliania lacunosa } & $\begin{array}{l}219-1-4 \\
219-1, \mathrm{CC} \\
(5-6)\end{array}$ & $\begin{array}{l}\text { T Globorotalia (Globorotalia) } \\
\text { ungulata }\end{array}$ & $\begin{array}{l}219-1-4 \\
219-1, \mathrm{CC} \\
(5-6)\end{array}$ & & \\
\hline & & $\begin{array}{l}\text { T Globorotalia (Truncorotalia) } \\
\text { truncatulinoides }\end{array}$ & $\begin{array}{l}219-1-4 \\
219-1, \mathrm{CC} \\
(5-6)\end{array}$ & & \\
\hline & & B Turborotalita pumilio & $\begin{array}{l}219-2-2 \\
219-2-3 \\
(8-9)\end{array}$ & 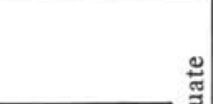 & \\
\hline & & $\begin{array}{l}\text { T Globorotalia (Globorotalia) } \\
\text { tumida subsp. } 1\end{array}$ & $\begin{array}{l}219-2-4 \\
219-2-5 \\
(11-12)\end{array}$ & 苞 & \\
\hline \multirow[t]{9}{*}{ T Scyphosphaera campanula } & $\begin{array}{l}219-2-3 \\
219-2, \mathrm{CC} \\
(9-13)\end{array}$ & $\mathrm{T}$ Globoquadrina pseudofoliata & $\begin{array}{l}219-2-5 \\
219-2, \mathrm{CC} \\
(12-13)\end{array}$ & $\sum_{\substack{0\\
}}^{0}$ & \\
\hline & & $\begin{array}{l}\mathrm{T} \text { Sphaeroidinella dehiscens } \\
\text { immatura }\end{array}$ & $\begin{array}{l}219-2-5 \\
219-2, \mathrm{CC} \\
(12-13) \\
\end{array}$ & 竎 & \\
\hline & & $\begin{array}{l}\text { B Globorotalia (Hirsutella) } \\
\text { theyeri }\end{array}$ & $\begin{array}{l}219-2, \mathrm{CC} \\
219-3-1 \\
(13-15) \\
\end{array}$ & $\underline{\mathscr{u}}$ & \\
\hline & & $\begin{array}{l}\text { T Turborotalia (Turborotalia) } \\
\text { humerosa trochoidea }\end{array}$ & $\begin{array}{l}219-3-3 \\
219-3-4 \\
(18-20)\end{array}$ & & \\
\hline & & $\begin{array}{l}\text { B Pulleniatina obliquiloculata } \\
\text { finalis }\end{array}$ & $\begin{array}{l}219-3-4 \\
219-3, \mathrm{CC} \\
(20-21)\end{array}$ & & \\
\hline & & $\begin{array}{l}\text { T Globorotalia (Truncorotalia) } \\
\text { tosaensis }\end{array}$ & $\begin{array}{l}219-3-4 \\
219-3, \mathrm{CC} \\
(20-21)\end{array}$ & & \\
\hline & & $\begin{array}{l}\text { T Turborotalia (Turborotalia) } \\
\text { humerosa humerosa }\end{array}$ & $\begin{array}{l}219-3-4 \\
219-3, \mathrm{CC} \\
(20-21)\end{array}$ & & \\
\hline & & $\begin{array}{l}\mathrm{T} \text { Globigerinoides obliquus } \\
\text { extremus }\end{array}$ & $\begin{array}{l}219-3, \mathrm{CC} \\
219-4-1 \\
(21-24) \\
\end{array}$ & & \\
\hline & & $\begin{array}{l}\text { T Globigerinoides quadrilobatus } \\
\text { fistulosus }\end{array}$ & $\begin{array}{l}219-4-2 \\
219-4-3 \\
(26-27)\end{array}$ & & \\
\hline
\end{tabular}


TABLE 1 - Continued

\begin{tabular}{|c|c|c|c|c|c|}
\hline \multicolumn{2}{|c|}{ Calcareous Nannofossils } & \multicolumn{2}{|l|}{ Foraminifera } & Radiolaria & \multirow[b]{2}{*}{$\begin{array}{l}\text { Interval: Site, } \\
\text { Core, Section, } \\
\text { Subbottom } \\
\text { Depth }(\mathrm{m})\end{array}$} \\
\hline Events & $\begin{array}{l}\text { Interval: Site, } \\
\text { Core, Section, } \\
\text { Subbottom } \\
\text { Depth (m) }\end{array}$ & Events & $\begin{array}{l}\text { Interval: Site, } \\
\text { Core, Section, } \\
\text { Subbottom } \\
\text { Depth (m) }\end{array}$ & Events & \\
\hline \multirow[t]{4}{*}{ T Discoaster brouweri } & $\begin{array}{l}219-4-4 \\
219-4-5 \\
(29-30)\end{array}$ & B Globigerina digitata & $\begin{array}{l}219-4-4 \\
219-4-5 \\
(29-30)\end{array}$ & & \\
\hline & & $\begin{array}{l}\text { B Globorotalia (Truncorotalia) } \\
\text { truncatulinoides }\end{array}$ & $\begin{array}{l}219-4-4 \\
219-4-5 \\
(29-30)\end{array}$ & & \\
\hline & & $\begin{array}{l}\text { T Globigerinoides obliquus } \\
\text { obliquus }\end{array}$ & $\begin{array}{l}219-4-4 \\
219-4-5 \\
(29-30)\end{array}$ & & \\
\hline & & $\begin{array}{l}\text { T Turborotalia (Turborotalia) } \\
\text { acostaensis acostaensis }\end{array}$ & $\begin{array}{l}219-4-4 \\
219-4-5 \\
(29-30)\end{array}$ & & \\
\hline \multirow[t]{7}{*}{ T Discoaster pentaradiatus } & $\begin{array}{l}219-4-5 \\
219-4, \mathrm{CC} \\
(30-32)\end{array}$ & $\begin{array}{l}\text { B "Globigerina" rubescens } \\
\text { tenella }\end{array}$ & $\begin{array}{l}219-4-5 \\
219-4-6 \\
(30-32)\end{array}$ & & \\
\hline & & $\begin{array}{l}\mathrm{T} \text { "Globigerina" rubescens } \\
\text { decoraperta }\end{array}$ & $\begin{array}{l}219-4-5 \\
219-4-6 \\
(30-32)\end{array}$ & & \\
\hline & & $\mathrm{T}$ Globigerinoides bollii & $\begin{array}{l}219-4-5 \\
219-4-6 \\
(30-32)\end{array}$ & 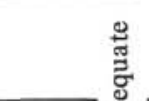 & \\
\hline & & $\begin{array}{l}\text { T Globorotalia (Globorotalia) } \\
\text { limbata }\end{array}$ & $\begin{array}{l}219-4-5 \\
219-4-6 \\
(30-32)\end{array}$ & 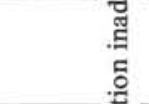 & \\
\hline & & T Orbulina suturalis & $\begin{array}{l}219-4-5 \\
219-4-6 \\
(30-32)\end{array}$ & 总 & \\
\hline & & $\begin{array}{l}\mathrm{T} \text { Pulleniatina obliquiloculata } \\
\text { praecursor }\end{array}$ & $\begin{array}{l}219-4-5 \\
219-4-6 \\
(30-32) \\
\end{array}$ & \multirow{2}{*}{ 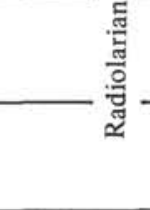 } & \\
\hline & & B Globigerina calida & $\begin{array}{l}219-4-6 \\
219-4, \mathrm{CC} \\
(32-32)\end{array}$ & & \\
\hline \multirow[t]{8}{*}{ T Discoaster surculus } & $\begin{array}{l}219-4, C C \\
219-5-3 \\
(32-36)\end{array}$ & $\begin{array}{l}\text { T Turborotalia (Turborotalia) } \\
\text { vincentae }\end{array}$ & $\begin{array}{l}219-4, C C \\
219-5-1 \\
(32-33)\end{array}$ & & \\
\hline & & $\begin{array}{l}\text { T Globorotalia (Globorotalia) } \\
\text { multicamerata }\end{array}$ & $\begin{array}{l}219-5-1 \\
219-5-2 \\
(33-35)\end{array}$ & & \\
\hline & & $\begin{array}{l}\text { T Sphaeroidinellopsis } \\
\text { sphaeroides }\end{array}$ & $\begin{array}{l}219-5-1 \\
219-5-2 \\
(33-35)\end{array}$ & & \\
\hline & & $\begin{array}{l}\text { B Globorotalia (Truncorotalia) } \\
\text { tosaensis }\end{array}$ & $\begin{array}{l}219-5-2 \\
219-5-3 \\
(35-36)\end{array}$ & & \\
\hline & & $\begin{array}{l}\text { T Globorotalia (Globorotalia) } \\
\text { merotumida }\end{array}$ & $\begin{array}{l}219-5-2 \\
219-5-3 \\
(35-36)\end{array}$ & & \\
\hline & & B Turborotalita pumilio & $\begin{array}{l}219-5-3 \\
219-5, \mathrm{CC} \\
(36-37)\end{array}$ & & \\
\hline & & $\begin{array}{l}\text { T Globoquadrina altispira } \\
\text { altispira }\end{array}$ & $\begin{array}{l}219-5-3 \\
219-5, \mathrm{CC} \\
(36-37)\end{array}$ & & \\
\hline & & $\begin{array}{l}\text { T Turborotalia (Turborotalia) } \\
\text { nigriniae }\end{array}$ & $\begin{array}{l}219-5-3 \\
219-5, \mathrm{CC} \\
(36-37)\end{array}$ & & \\
\hline
\end{tabular}


TABLE 1 - Continued

\begin{tabular}{|c|c|c|c|c|c|}
\hline \multicolumn{2}{|c|}{ Calcareous Nannofossils } & \multicolumn{2}{|l|}{ Foraminifera } & \multicolumn{2}{|c|}{ Radiolaria } \\
\hline Events & $\begin{array}{l}\text { Interval: Site, } \\
\text { Core, Section, } \\
\text { Subbottom } \\
\text { Depth (m) }\end{array}$ & Events & $\begin{array}{l}\text { Interval: Site, } \\
\text { Core, Section, } \\
\text { Subbottom } \\
\text { Depth }(\mathrm{m})\end{array}$ & Events & $\begin{array}{l}\text { Interval: Site } \\
\text { Core, Section, } \\
\text { Subbottom, } \\
\text { Depth (m) }\end{array}$ \\
\hline \multirow[t]{8}{*}{$\mathrm{T}$ Sphenolithus abies } & $\begin{array}{l}219-5, \mathrm{CC} \\
219-6-2 \\
(37-44)\end{array}$ & $\begin{array}{l}\text { B Turborotalia (Turborotalia) } \\
\text { pseudopima }\end{array}$ & $\begin{array}{l}219-5, \mathrm{CC} \\
219-6-1 \\
(37-42) \\
\end{array}$ & & \\
\hline & & $\begin{array}{l}\mathrm{T} \text { Globoquadrina larmeui } \\
\text { obesa }\end{array}$ & $\begin{array}{l}219-5, \mathrm{CC} \\
219-6-1 \\
(37-42)\end{array}$ & & \\
\hline & & $\begin{array}{l}\text { B Globigerinoides } \\
\text { quadrilobatus fistulosus }\end{array}$ & $\begin{array}{l}219-6-1 \\
219-6-2 \\
(42-44)\end{array}$ & & \\
\hline & & B Globoquadrina pseudofoliata & $\begin{array}{l}219-6-1 \\
219-6-2 \\
(42-44)\end{array}$ & & \\
\hline & & $\begin{array}{l}\text { B Pulleniatina obliquiloculata } \\
\text { obliquiloculata }\end{array}$ & $\begin{array}{l}219-6-1 \\
219-6-2 \\
(42-44)\end{array}$ & & \\
\hline & & $\begin{array}{l}\text { B Sphaeroidinella dehiscens } \\
\text { dehiscens }\end{array}$ & $\begin{array}{l}219-6-2 \\
219-6-2 \\
(42-44)\end{array}$ & & \\
\hline & & $\mathrm{T}$ Globorotaloides variabilis & $\begin{array}{l}219-6-1 \\
219-6-2 \\
(42-44)\end{array}$ & & \\
\hline & & $\begin{array}{l}\text { T Sphaeroidinellopsis } \\
\text { subdehiscens }\end{array}$ & $\begin{array}{l}219-6-1 \\
219-6-2 \\
(42-44) \\
\end{array}$ & 䒕 & \\
\hline \multirow[t]{11}{*}{$\begin{array}{l}\text { T Reticulofenestra } \\
\text { pseudoumbilica }\end{array}$} & $\begin{array}{l}219-6-2 \\
219-6-3 \\
(44-45)\end{array}$ & $\begin{array}{l}\text { B "Globigerina" rubescens } \\
\text { rubescens }\end{array}$ & $\begin{array}{l}219-6-2 \\
219-6-3 \\
(44-45)\end{array}$ & 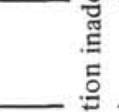 & \\
\hline & & B Globoquadrina conglomerata & $\begin{array}{l}219-6-2 \\
219-6-3 \\
(44-45)\end{array}$ & 总 & \\
\hline & & $\begin{array}{l}\text { B Globorotalia (Truncorotalia) } \\
\text { crassaformis s.1. }\end{array}$ & $\begin{array}{l}219-6-2 \\
219-6-3 \\
(44-45) \\
\end{array}$ & 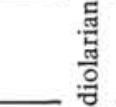 & \\
\hline & & $\begin{array}{l}\text { B Sphaeroidinella dehiscens } \\
\text { immatura }\end{array}$ & $\begin{array}{l}219-6-2 \\
219-6-3 \\
(44-45)\end{array}$ & $\cong$ & \\
\hline & & $\begin{array}{l}\text { B Turborotalia } \\
\text { (Neogloboquadrina) dutertrei }\end{array}$ & $\begin{array}{l}219-6-2 \\
219-6-3 \\
(44-45)\end{array}$ & & \\
\hline & & $\begin{array}{l}\text { T Globigerinoides conglobatus } \\
\text { canimarensis }\end{array}$ & $\begin{array}{l}219-6-2 \\
219-6-3 \\
(44-45) \\
\end{array}$ & & \\
\hline & & $\begin{array}{l}\mathrm{T} \text { Globorotalia (Hirsutella) } \\
\text { margaritae }\end{array}$ & $\begin{array}{l}219-6-2 \\
219-6-3 \\
(44-45) \\
\end{array}$ & & \\
\hline & & $\begin{array}{l}\text { T Globorotalia (Hirsutella) } \\
\text { hirsuta praehirsuta }\end{array}$ & $\begin{array}{l}219-6-2 \\
219-6-3 \\
(44-45) \\
\end{array}$ & & \\
\hline & & $\mathrm{T}$ Pulleniatina primalis & $\begin{array}{l}219-6-2 \\
219-6-3 \\
(44-45) \\
\end{array}$ & & \\
\hline & & $\begin{array}{l}\text { T Sphaeroidinellopsis } \\
\text { seminulina }\end{array}$ & $\begin{array}{l}219-6-2 \\
219-6-3 \\
(44-45) \\
\end{array}$ & & \\
\hline & & $\begin{array}{l}\mathrm{T} \text { Globoquadrina dehiscens } \\
\text { dehiscens }\end{array}$ & $\begin{array}{l}219-6-3 \\
219-6-4 \\
(45-47) \\
\end{array}$ & & \\
\hline
\end{tabular}


TABLE 1 - Continued

\begin{tabular}{|c|c|c|c|c|c|}
\hline \multicolumn{2}{|c|}{ Calcareous Nannofossils } & \multicolumn{2}{|l|}{ Foraminifera } & \multicolumn{2}{|c|}{ Radiolaria } \\
\hline Events & $\begin{array}{l}\text { Interval: Site, } \\
\text { Core, Section } \\
\text { Subbottom } \\
\text { Depth (m) }\end{array}$ & Events & $\begin{array}{l}\text { Interval: Site, } \\
\text { Core, Section, } \\
\text { Subbottom } \\
\text { Depth (m) }\end{array}$ & Events & $\begin{array}{l}\text { Interval: Site, } \\
\text { Core, Section, } \\
\text { Subbottom } \\
\text { Depth (m) }\end{array}$ \\
\hline & & $\begin{array}{l}\mathrm{T} \text { Sphaeroidinellopsis } \\
\text { paenedehiscens }\end{array}$ & $\begin{array}{l}219-6-3 \\
219-6-4 \\
(45-47)\end{array}$ & & \\
\hline & & $\begin{array}{l}\text { T Turborotalia (Turborotalia) } \\
\text { humerosa praepulleniatina }\end{array}$ & $\begin{array}{l}219-6-3 \\
219-6-4 \\
(45-47)\end{array}$ & & \\
\hline & & $\begin{array}{l}\text { B Globorotalia (Globorotalia) } \\
\text { tumida subsp. } 1\end{array}$ & $\begin{array}{l}219-6-4 \\
219-6-5 \\
(47-48)\end{array}$ & & \\
\hline & & $\begin{array}{l}\text { B Turborotalia (Turborotalia) } \\
\text { humerosa trochoidea }\end{array}$ & $\begin{array}{l}219-6-4 \\
219-6-5 \\
(47-48)\end{array}$ & & \\
\hline & & B Globigerinoides ruber & $\begin{array}{l}219-6-5 \\
219-6-6 \\
(48-50)\end{array}$ & & \\
\hline & & T "Globigerina" nepenthes & $\begin{array}{l}219-6-5 \\
219-6-6 \\
(48-50)\end{array}$ & & \\
\hline \multirow[t]{13}{*}{$\begin{array}{ll}\mathrm{T} & \text { Ceratolithus } \\
\text { tricorniculatus }\end{array}$} & $\begin{array}{l}219-6-6 \\
219-6, \mathrm{CC} \\
(50-51)\end{array}$ & $\begin{array}{l}\mathrm{T} \text { Globigerinella siphonifera } \\
\text { involuta }\end{array}$ & $\begin{array}{l}219-6-6 \\
219-6, \mathrm{CC} \\
(50-51)\end{array}$ & 兽 & \\
\hline & & $\begin{array}{l}\mathrm{T} \text { Globorotalia (Globorotalia) } \\
\text { tumida plesiotumida }\end{array}$ & $\begin{array}{l}219-6-6 \\
219-6, \mathrm{CC} \\
(50-51)\end{array}$ & . & \\
\hline & & $\begin{array}{l}\text { B Pulleniatina obliquiloculata } \\
\text { praecursor }\end{array}$ & $\begin{array}{l}219-6, \mathrm{CC} \\
219-7-1 \\
(51-51)\end{array}$ & 总 & \\
\hline & & $\begin{array}{l}\text { B Sphaeroidinellopsis } \\
\text { sphaeroides }\end{array}$ & $\begin{array}{l}219-6, \mathrm{CC} \\
219-7-1 \\
(51-51)\end{array}$ & 爱 & \\
\hline & & B Streptochilus tokelauae & $\begin{array}{l}219-7-1 \\
219-7-2 \\
(51-53)\end{array}$ & & \\
\hline & & $\begin{array}{l}\text { T Globigerina } \\
\text { angustiumbilicata }\end{array}$ & $\begin{array}{l}219-7-1 \\
219-7-2 \\
(51-53)\end{array}$ & & \\
\hline & & $\begin{array}{l}\text { B Globorotalia (Globorotalia) } \\
\text { ungulata }\end{array}$ & $\begin{array}{l}219-7-2 \\
219-7-3 \\
(53-54)\end{array}$ & & \\
\hline & & $\begin{array}{l}\text { B Turborotalia (Turborotalia) } \\
\text { nigriniae }\end{array}$ & $\begin{array}{l}219-7-2 \\
219-7-3 \\
(53-54)\end{array}$ & & \\
\hline & & B Turborotalita humilis & $\begin{array}{l}219-7-3 \\
219-7-4 \\
(54-56)\end{array}$ & & \\
\hline & & T Streptochilus globigerum & $\begin{array}{l}219-7-3 \\
219-7-4 \\
(54-56)\end{array}$ & & \\
\hline & & $\begin{array}{l}\text { B Globorotalia (Globorotalia) } \\
\text { multicamerata }\end{array}$ & $\begin{array}{l}219-7-4 \\
219-7-5 \\
(56-57)\end{array}$ & & \\
\hline & & B Temuitella anfracta & $\begin{array}{l}219-7-4 \\
219-7-5 \\
(56-57)\end{array}$ & & \\
\hline & & $\begin{array}{l}\text { B Globorotalia (Hirsutella) } \\
\text { hirsuta praehirsuta }\end{array}$ & $\begin{array}{l}219-7-5 \\
219-7-6 \\
(57-59)\end{array}$ & & \\
\hline
\end{tabular}


TABLE 1 - Continued

\begin{tabular}{|c|c|c|c|c|c|}
\hline \multicolumn{2}{|l|}{ Calcareous Nannofossils } & \multicolumn{2}{|l|}{ Foraminifera } & \multicolumn{2}{|c|}{ Radiolaria } \\
\hline Eyents & $\begin{array}{l}\text { Interval: Site, } \\
\text { Core, Section, } \\
\text { Subbottom } \\
\text { Depth }(\mathrm{m})\end{array}$ & Events & $\begin{array}{l}\text { Interval: Site, } \\
\text { Core, Section, } \\
\text { Subbottom } \\
\text { Depth (m) }\end{array}$ & Events & $\begin{array}{l}\text { Interval: Site, } \\
\text { Core, Section, } \\
\text { Subbottom } \\
\text { Depth }(m)\end{array}$ \\
\hline & & $\begin{array}{l}\text { B Globigerinita glutinata } \\
\text { parkerae }\end{array}$ & $\begin{array}{l}219-7-6 \\
219-7, \mathrm{CC} \\
(59-59)\end{array}$ & & \\
\hline & & $\begin{array}{l}\text { B Globorotalia (Hirsutella) } \\
\text { margaritae }\end{array}$ & $\begin{array}{l}219-7-6 \\
219-7, \mathrm{CC} \\
(59-59)\end{array}$ & & \\
\hline & & $\begin{array}{l}\text { B Globigerinoides conglobatus } \\
\text { conglobatus }\end{array}$ & $\begin{array}{l}219-8-2 \\
219-8-3 \\
(62-63)\end{array}$ & & \\
\hline & & $\begin{array}{l}\text { B Globorotalia (Globorotalia) } \\
\text { tumida flexuosa }\end{array}$ & $\begin{array}{l}219-8-2 \\
219-8-3 \\
(62-63)\end{array}$ & & \\
\hline & & $\begin{array}{l}\text { T Turborotalia (Turborotalia) } \\
\text { acostaensis tegillata }\end{array}$ & $\begin{array}{l}219-8-2 \\
219-8-3 \\
(62-63)\end{array}$ & & \\
\hline & & $\mathrm{T}$ Candeina nitida praenitida & $\begin{array}{l}219-8-3 \\
219-8-4 \\
(63-65)\end{array}$ & & \\
\hline & & $\mathrm{T}$ Pulleniatina praespectabilis & $\begin{array}{l}219-8-4 \\
219-8-5 \\
(63-65)\end{array}$ & & \\
\hline \multirow[t]{2}{*}{$\mathrm{T}$ Discoaster quinqueramus } & $\begin{array}{l}219-8-5 \\
219-8-6 \\
(63-65)\end{array}$ & $\begin{array}{l}\text { B Globorotalia (Globorotalia) } \\
\text { tumida tumida }\end{array}$ & $\begin{array}{l}219-8-5 \\
219-8-6 \\
(63-65)\end{array}$ & \multicolumn{2}{|l|}{ 氶 } \\
\hline & & T Streptochilus latum & $\begin{array}{l}219-9-1 \\
219-9-2 \\
(69-71) \\
\end{array}$ & 祃 & \\
\hline \multirow[t]{8}{*}{ T Discoaster hamatus } & $\begin{array}{l}219-9-4 \\
219-9-5 \\
(74-75)\end{array}$ & $\mathrm{T}$ "Slobigerina" bulbosa & $\begin{array}{l}219-9-4 \\
219-9-5 \\
(74-75)\end{array}$ & . & \\
\hline & & B Pulleniatina praespectabilis & $\begin{array}{l}219-9-5 \\
219-9-6 \\
(75-77) \\
\end{array}$ & \multirow[t]{2}{*}{ 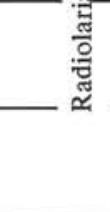 } & \\
\hline & & $\begin{array}{l}\text { B Turborotalia (Turborotalia) } \\
\text { vincentae }\end{array}$ & $\begin{array}{l}219-10-1 \\
219-10-2 \\
(78-80) \\
\end{array}$ & & \\
\hline & & B Streptochilus globigerum & $\begin{array}{l}219-10-2 \\
219-10-3 \\
(80-81)\end{array}$ & & \\
\hline & & $\begin{array}{l}\text { B Sphaeroidinellopsis } \\
\text { paenedehiscens }\end{array}$ & $\begin{array}{l}219-10-4 \\
219-10-5 \\
(81-82)\end{array}$ & & \\
\hline & & $\begin{array}{l}\text { B Turborotalia (Turborotalia) } \\
\text { humerosa praepulleniatina }\end{array}$ & $\begin{array}{l}219-10-4 \\
219-10-5 \\
(81-82)\end{array}$ & & \\
\hline & & B Pulleniatina primalis & \begin{tabular}{|l|}
$219-10-6$ \\
$219-10, \mathrm{CC}$ \\
$(81-82)$ \\
\end{tabular} & & \\
\hline & & B Streptochilus latum & $\begin{array}{l}219-11-2 \\
219-11-3 \\
(84-85)\end{array}$ & & \\
\hline \multirow[t]{2}{*}{$\mathrm{T}$ Discoaster dilatus } & $\begin{array}{l}219-11-3 \\
219-11-4 \\
(85-87)\end{array}$ & & & & \\
\hline & & B Globorotaloides hexagonus & $\begin{array}{l}219-11-4 \\
219-11-5 \\
(87-88)\end{array}$ & & \\
\hline
\end{tabular}


TABLE 1 - Continued

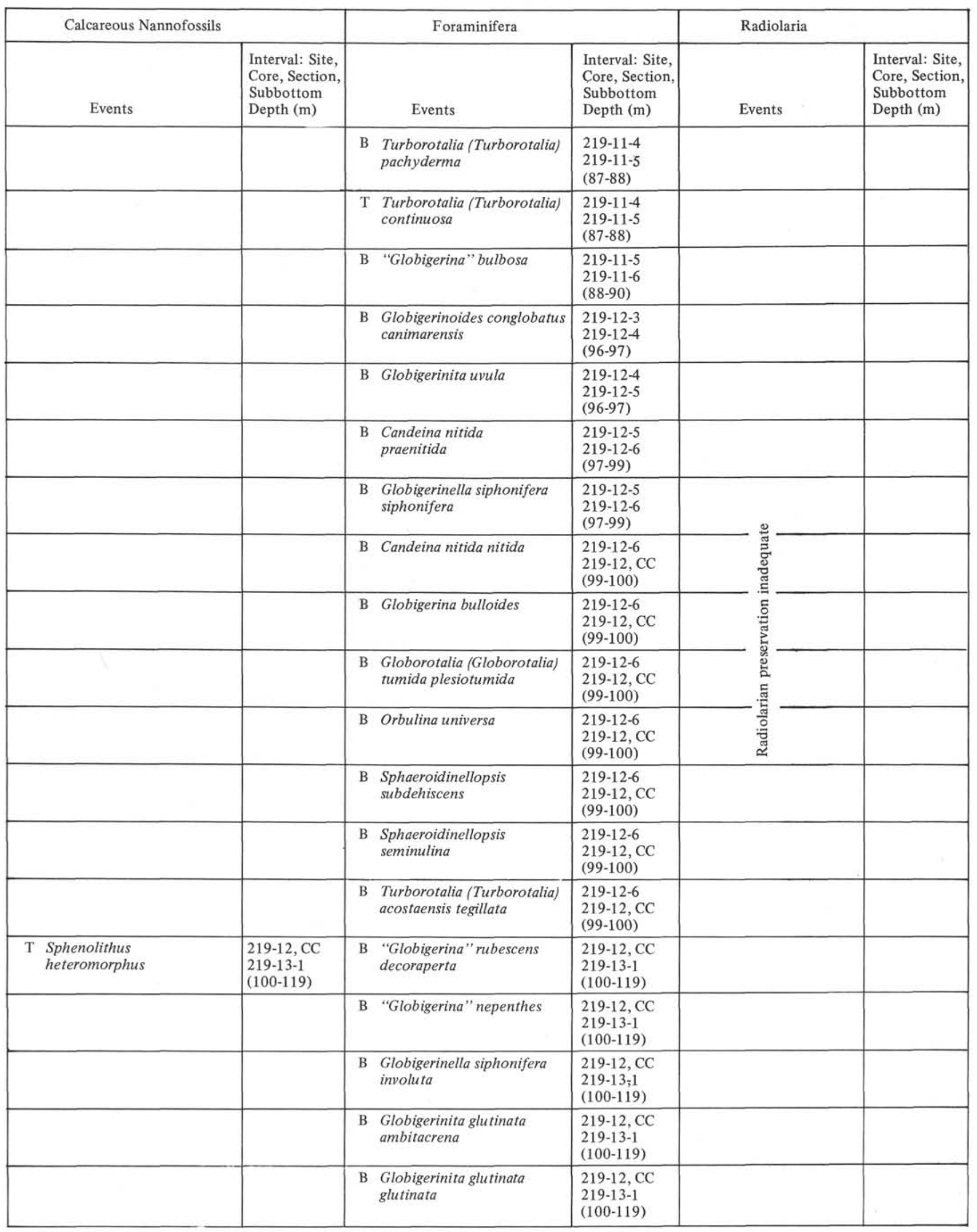


TABLE 1 - Continued

\begin{tabular}{|c|c|c|c|c|c|}
\hline \multicolumn{2}{|c|}{ Calcareous Nannofossils } & \multicolumn{2}{|l|}{ Foraminifera } & \multicolumn{2}{|l|}{ Radiolaria } \\
\hline Events & $\begin{array}{l}\text { Interval, Site, } \\
\text { Core, Section, } \\
\text { Subbottom } \\
\text { Depth (m) }\end{array}$ & Events & $\begin{array}{l}\text { Interval, Site, } \\
\text { Core, Section, } \\
\text { Subbottom } \\
\text { Depth (m) }\end{array}$ & Events & $\begin{array}{l}\text { Interval, Site, } \\
\text { Core, Section, } \\
\text { Subbottom } \\
\text { Depth (m) }\end{array}$ \\
\hline & & $\begin{array}{l}\text { B Globigerinoides obliquus } \\
\text { extremus }\end{array}$ & $\begin{array}{l}219-12, \mathrm{CC} \\
219-13-1 \\
(100-119) \\
\end{array}$ & & \\
\hline & & $\begin{array}{l}\text { B Globigerinoides } \\
\text { quadrilobatus sacculifer }\end{array}$ & $\begin{array}{l}219-12, \mathrm{CC} \\
219-13-1 \\
(100-119) \\
\end{array}$ & & \\
\hline & & B Globigerinoides bollii & $\begin{array}{l}219-12, \mathrm{CC} \\
219-13-1 \\
(100-119)\end{array}$ & & \\
\hline & & $\begin{array}{l}\text { B Globorotalia (Globorotalia) } \\
\text { cultrata }\end{array}$ & $\begin{array}{l}219-12, \mathrm{CC} \\
219-13,1 \\
(100-119)\end{array}$ & & \\
\hline & & $\begin{array}{l}\text { B Globorotalia (Globorotalia) } \\
\text { limbata }\end{array}$ & $\begin{array}{l}219-12, \mathrm{CC} \\
219-13-1 \\
(100-119) \\
\end{array}$ & & \\
\hline & & $\begin{array}{l}\text { B Globorotalia (Globorotalia) } \\
\text { merotumida }\end{array}$ & $\begin{array}{l}219-12, \mathrm{CC} \\
219-13-1 \\
(100-119) \\
\end{array}$ & & \\
\hline & & $\begin{array}{l}\text { B Globorotalia (Hirsutella) } \\
\text { scitula scitula }\end{array}$ & $\begin{array}{l}219-12, \mathrm{CC} \\
219-13-1 \\
(100-119)\end{array}$ & $\underset{\overparen{J}}{\stackrel{\Xi}{\Xi}}$ & \\
\hline & & $\begin{array}{l}\text { B Turborotalia (Turborotalia) } \\
\text { humerosa humerosa }\end{array}$ & $\begin{array}{l}219-12, \mathrm{CC} \\
219-13-1 \\
(100-119) \\
\end{array}$ & 荧 & \\
\hline & & $\begin{array}{l}\text { B Turborotalia (Turborotalia) } \\
\text { acostaensis acostaensis }\end{array}$ & $\begin{array}{l}219-12, C C \\
219-13-1 \\
(100-119)\end{array}$ & 劳 & \\
\hline & & $\begin{array}{l}\text { B Turborotalia } \\
\text { "quinqueloba" }\end{array}$ & $\begin{array}{l}219-12, \mathrm{CC} \\
219-13-1 \\
(100-119) \\
\end{array}$ & 裿 & \\
\hline & & T Clavatorella bermudezi & $\begin{array}{l}219-12, \mathrm{CC} \\
219-13-1 \\
(100-119) \\
\end{array}$ & $\stackrel{\widetilde{z}}{\simeq}$ & \\
\hline & & T Globigerinita boweni & $\begin{array}{l}219-12, \mathrm{CC} \\
219-13-1 \\
(100-119) \\
\end{array}$ & & \\
\hline & & $\begin{array}{l}\text { T Globigerinoides } \\
\text { subquadratus }\end{array}$ & $\begin{array}{l}219-12, \mathrm{CC} \\
219-13-1 \\
(100-119) \\
\end{array}$ & & \\
\hline & & $\begin{array}{l}\mathrm{T} \text { Globorotalia (Fohsella) } \\
\text { peripheroronda }\end{array}$ & $\begin{array}{l}219-12, \mathrm{CC} \\
219-13-1 \\
(100-119)\end{array}$ & & \\
\hline & & $\begin{array}{l}\mathrm{T} \text { Globorotalia (Fohsella) } \\
\text { peripheroacuta }\end{array}$ & $\begin{array}{l}219-12, \mathrm{CC} \\
219-13-1 \\
(100-119)\end{array}$ & & \\
\hline & & $\begin{array}{l}\mathrm{T} \text { Globorotalia (Globorotalia) } \\
\text { praemenardii }\end{array}$ & $\begin{array}{l}219-12, \mathrm{CC} \\
219-13-1 \\
(100-119) \\
\end{array}$ & & \\
\hline & & $\mathrm{T}$ Tenuitella clemenciae & $\begin{array}{l}219-12, \mathrm{CC} \\
219-13-1 \\
(100-119)\end{array}$ & & \\
\hline & & $\begin{array}{l}\text { T Turborotalia (Turborotalia) } \\
\text { siakensis }\end{array}$ & $\begin{array}{l}219-12, C C \\
219-13-1 \\
(100-119) \\
\end{array}$ & & \\
\hline & & $\begin{array}{l}\mathrm{T} \text { Turborotalia (Turborotalia) } \\
\text { mayeri }\end{array}$ & $\begin{array}{l}219-12, \mathrm{CC} \\
219-13-1 \\
(100-119)\end{array}$ & & \\
\hline
\end{tabular}


TABLE 1 - Continued

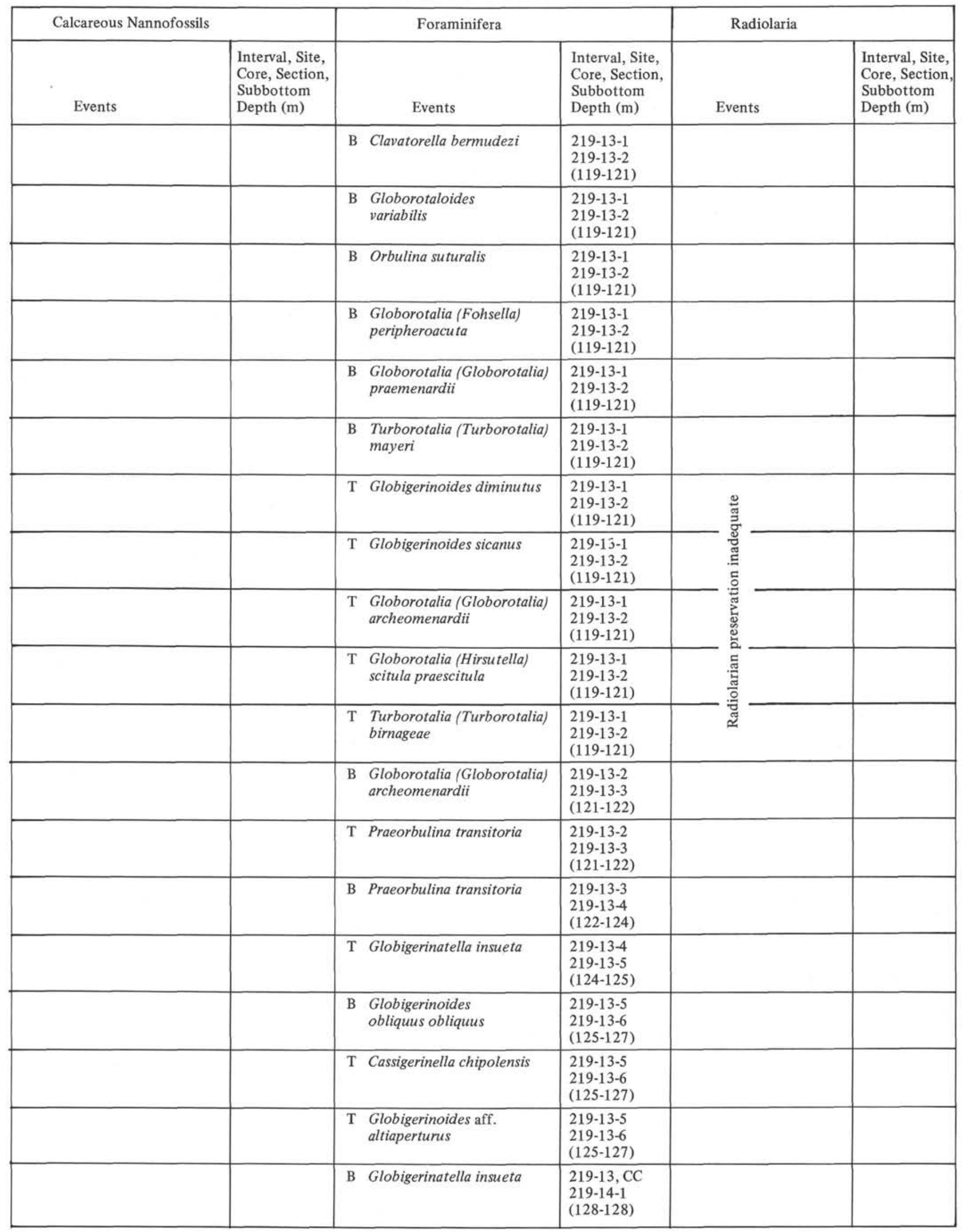


TABLE 1 - Continued

\begin{tabular}{|c|c|c|c|c|c|c|}
\hline \multicolumn{3}{|c|}{ Calcareous Nannofossils } & \multicolumn{2}{|l|}{ Foraminifera } & \multicolumn{2}{|l|}{ Radiolaria } \\
\hline \multicolumn{2}{|l|}{ Events } & $\begin{array}{l}\text { Interval, Site, } \\
\text { Core, Section, } \\
\text { Subbottom } \\
\text { Depth }(\mathrm{m})\end{array}$ & Events & $\begin{array}{l}\text { Interval, Site, } \\
\text { Core, Section, } \\
\text { Subbottom } \\
\text { Depth (m) }\end{array}$ & Events & $\begin{array}{l}\text { Interval, Site } \\
\text { Core, Section, } \\
\text { Subbottom, } \\
\text { Depth (m) }\end{array}$ \\
\hline & & & T “Globigerina” pseudodruryi & $\begin{array}{l}219-13, \mathrm{CC} \\
219-14-1 \\
(128-128) \\
\end{array}$ & & \\
\hline \multirow{8}{*}{\multicolumn{2}{|c|}{$\begin{array}{l}\text { T Helicopontosphaera } \\
\text { ampliaperta }\end{array}$}} & \begin{tabular}{|l|}
$219-14-1$ \\
$219-14-2$ \\
$(128-130)$ \\
\end{tabular} & $\begin{array}{l}\mathrm{T} \text { Globoquadrina dehiscens } \\
\text { praedehiscens }\end{array}$ & \begin{tabular}{|l|}
$219-14-1$ \\
$219-14-2$ \\
$(128-130)$ \\
\end{tabular} & & \\
\hline & & & T Globorotaloides suteri & $\begin{array}{l}219-14-1 \\
219-14-2 \\
(128-130)\end{array}$ & & \\
\hline & & & B "Globigerina" pseudodruryi & $\begin{array}{l}219-14-3 \\
219-14-4 \\
(131-133)\end{array}$ & & \\
\hline & & & B Globigerinoides subquadratus & \begin{tabular}{|l|}
$219-14-3$ \\
$219-14-4$ \\
$(131-133)$ \\
\end{tabular} & & \\
\hline & & & B Globigerinoides diminutus & $\begin{array}{l}219-14-3 \\
219-14-4 \\
(131-133)\end{array}$ & & \\
\hline & & & B Tenuitella clemenciae & $\begin{array}{l}219-14-4 \\
219-14-5 \\
(133-134)\end{array}$ & & \\
\hline & & & $\begin{array}{l}\text { B Globoquadrina larmeui } \\
\text { obesa }\end{array}$ & \begin{tabular}{|l|}
$219-14-5$ \\
$219-14-6$ \\
$(134-136)$ \\
\end{tabular} & & \\
\hline & & & $\begin{array}{l}\text { B Turborotalia (Turborotalia) } \\
\text { continuosa }\end{array}$ & \begin{tabular}{|l|}
$219-14-5$ \\
$219-14-6$ \\
$(134-136)$ \\
\end{tabular} & & \\
\hline \multirow[t]{10}{*}{$\begin{array}{l}\text { T Sphenolithus } \\
\text { predistentus }\end{array}$} & $\begin{array}{l}220-8, \mathrm{CC} \\
220-9-1 \\
(117-150) \\
\end{array}$ & \begin{tabular}{|l|}
$219-14, \mathrm{CC}$ \\
$219-15-1$ \\
$(136-156)$ \\
\end{tabular} & B Globigerinita boweni & \begin{tabular}{|l|}
$219-14, \mathrm{CC}$ \\
$219-15-1$ \\
$(136-156)$ \\
\end{tabular} & $\begin{array}{l}\text { Petalospyris triceros } \\
\rightarrow \text { Dorcadospyris } \\
\text { ateuchus }\end{array}$ & $\begin{array}{l}220-8, \mathrm{CC} \\
220-9-1 \\
(117-150) \\
\end{array}$ \\
\hline & & & $\begin{array}{l}\text { B Globigerinoides } \\
\text { quadrilobatus quadrilobatus }\end{array}$ & \begin{tabular}{|l|}
$219-14, \mathrm{CC}$ \\
$219-15-1$ \\
$(136-156)$ \\
\end{tabular} & & \\
\hline & & & B Globigerinoides sicanus & \begin{tabular}{|l|}
$219-14, \mathrm{CC}$ \\
$219-15-1$ \\
$(136-156)$ \\
\end{tabular} & & \\
\hline & & & $\begin{array}{l}\text { B Globigerinoides aff. } \\
\text { altiaperturus }\end{array}$ & \begin{tabular}{|l|}
$219-14, \mathrm{CC}$ \\
$219-15-1$ \\
$(136-156)$ \\
\end{tabular} & & \\
\hline & & & $\begin{array}{l}\text { B Globoquadrina altispira } \\
\text { altispira }\end{array}$ & \begin{tabular}{|l|}
$219-14, \mathrm{CC}$ \\
$219-15-1$ \\
$(136-156)$ \\
\end{tabular} & & \\
\hline & & & $\begin{array}{l}\text { B Globoquadrina } \\
\text { venezuelana }\end{array}$ & \begin{tabular}{|l|}
$219-14, \mathrm{CC}$ \\
$219-15-1$ \\
$(136-156)$ \\
\end{tabular} & & \\
\hline & & & $\begin{array}{l}\text { B Globoquadrina dehiscens } \\
\text { dehiscens }\end{array}$ & \begin{tabular}{|l|}
$219-14, \mathrm{CC}$ \\
$219-15-1$ \\
$(136-156)$ \\
\end{tabular} & & \\
\hline & & & $\begin{array}{l}\text { B Globoquadrina dehiscens } \\
\text { praedehiscens }\end{array}$ & \begin{tabular}{|l|}
$219-14, \mathrm{CC}$ \\
$219-15-1$ \\
$(136-156)$ \\
\end{tabular} & & \\
\hline & & & $\begin{array}{l}\text { B Globorotalia (Fohsella) } \\
\text { peripheroronda }\end{array}$ & \begin{tabular}{|l|}
$219-14, \mathrm{CC}$ \\
$219-15-1$ \\
$(136-156)$ \\
\end{tabular} & & \\
\hline & & & $\begin{array}{l}\text { B Globorotalia (Hirsutella) } \\
\text { scitula praescitula }\end{array}$ & $\begin{array}{l}219-14, C C \\
219-15-1 \\
(136-156)\end{array}$ & & \\
\hline
\end{tabular}


TABLE 1 - Continued

\begin{tabular}{|c|c|c|c|c|c|}
\hline \multicolumn{2}{|c|}{ Calcareous Nannofossils } & \multicolumn{2}{|l|}{ Foraminifera } & \multicolumn{2}{|l|}{ Radiolaria } \\
\hline Events & $\begin{array}{l}\text { Interval, Site, } \\
\text { Core, Section, } \\
\text { Subbottom } \\
\text { Depth (m) }\end{array}$ & Events & $\begin{array}{l}\text { Interval, Site, } \\
\text { Core, Section, } \\
\text { Subbottom } \\
\text { Depth }(\mathrm{m})\end{array}$ & Events & $\begin{array}{l}\text { Interval, Site, } \\
\text { Core, Section, } \\
\text { Subbottom } \\
\text { Depth (m) }\end{array}$ \\
\hline & & $\begin{array}{l}\text { B Turborotalia (Turborotalia) } \\
\text { siakensis }\end{array}$ & $\begin{array}{l}219-14, \mathrm{CC} \\
219-15-1 \\
(136-156) \\
\end{array}$ & & \\
\hline & & $\begin{array}{l}\text { B Turborotalia (Turborotalia) } \\
\text { birnageae }\end{array}$ & $\begin{array}{l}219-14, \mathrm{CC} \\
219-15-1 \\
(136-156)\end{array}$ & & \\
\hline & & T Catapsydrax perus & $\begin{array}{l}219-14, \mathrm{CC} \\
219-15-1 \\
(136-156)\end{array}$ & & \\
\hline & & T Chiloguembelina cubensis & $\begin{array}{l}219-14, \mathrm{CC} \\
219-15-1 \\
(136-156)\end{array}$ & & \\
\hline & & T Globoquadrina galavisi & $\begin{array}{l}219-14, \mathrm{CC} \\
219-15-1 \\
(136-156)\end{array}$ & & \\
\hline & & $\begin{array}{l}\mathrm{T} \text { Globoquadrina tripartita } \\
\text { tapuriensis }\end{array}$ & $\begin{array}{l}219-14, \mathrm{CC} \\
219-15-1 \\
(136-156)\end{array}$ & & \\
\hline & & $\begin{array}{l}\text { T Globoquadrina tripartita } \\
\text { tripartita }\end{array}$ & $\begin{array}{l}219-14, \mathrm{CC} \\
219-15-1 \\
(136-156)\end{array}$ & & \\
\hline & & $\begin{array}{l}\mathrm{T} \text { Pseudohastigerina } \\
\text { naguewichiensis } \\
\text { barbadoensis }\end{array}$ & $\begin{array}{l}219-14, \mathrm{CC} \\
219-15-1 \\
(136-156)\end{array}$ & & \\
\hline & & T Subbotina winkleri & $\begin{array}{l}219-14, \mathrm{CC} \\
219-15-1 \\
(136-156)\end{array}$ & & \\
\hline & & $\begin{array}{l}\text { T Turborotalia (Turborotalia) } \\
\text { opima nana }\end{array}$ & $\begin{array}{l}219-14, \mathrm{CC} \\
219-15-1 \\
(136-156) \\
\end{array}$ & & \\
\hline \multirow[t]{6}{*}{$\begin{array}{l}\mathrm{T} \text { Helicopontosphaera } \\
\text { truncata }\end{array}$} & $\begin{array}{l}220-10-2 \\
220-10, \mathrm{CC} \\
(161-162)\end{array}$ & $\begin{array}{l}\mathrm{T} \text { Turborotalia (Turborotalia) } \\
\text { prasaepis }\end{array}$ & $\begin{array}{l}219-14, \mathrm{CC} \\
219-15-1 \\
(136-156)\end{array}$ & & \\
\hline & & T Subbotina angiporoides & $\begin{array}{l}219-15-1 \\
219-15-2 \\
(156-158)\end{array}$ & & \\
\hline & & $\mathrm{T}$ Tenuitella gemma & $\begin{array}{l}219-15-1 \\
219-15-2 \\
(156-158)\end{array}$ & & \\
\hline & & $\begin{array}{l}\mathrm{T} \text { Catapsydrax unicavus } \\
\text { primitivus }\end{array}$ & $\begin{array}{l}219-15-2 \\
219-15-3 \\
(158-159)\end{array}$ & & \\
\hline & & $\begin{array}{l}\text { T Turborotalia (Turborotalia) } \\
\text { ampliapertura }\end{array}$ & $\begin{array}{l}219-15-2 \\
219-15-3 \\
(158-159)\end{array}$ & & \\
\hline & & $\begin{array}{l}\text { T Turborotalia (Turborotalia) } \\
\text { pseudoampliapertura }\end{array}$ & $\begin{array}{l}219-15-2 \\
219-15-3 \\
(158-159)\end{array}$ & & \\
\hline $\begin{array}{l}\text { T Reticulofenestra } \\
\text { umbilica }\end{array}$ & $\begin{array}{l}219-15, \mathrm{CC} \\
219-16-1 \\
(165-165)\end{array}$ & & & $\mathrm{T}$ Theocampe pirum & $\begin{array}{l}220-10-1 \\
220-10-2 \\
(159-161)\end{array}$ \\
\hline \multirow[t]{2}{*}{$\begin{array}{l}\mathrm{T} \text { Helicopontosphaera } \\
\text { reticulata }\end{array}$} & $\begin{array}{l}219-15, \mathrm{CC} \\
219-16-1 \\
(165-165)\end{array}$ & & & & \\
\hline & & $\begin{array}{l}\text { B Cassigerinella } \\
\text { chipolensis }\end{array}$ & $\begin{array}{l}219-16-1 \\
219-16-2 \\
(165-167)\end{array}$ & & \\
\hline
\end{tabular}


C. NIGRINI, R. FLEISHER, J. BOUDREAUX

TABLE 1 - Continued

\begin{tabular}{|c|c|c|c|c|c|c|}
\hline \multicolumn{3}{|c|}{ Calcareous Nannofossils } & \multicolumn{2}{|l|}{ Foraminifera } & \multicolumn{2}{|l|}{ Radiolaria } \\
\hline \multicolumn{2}{|l|}{ Events } & $\begin{array}{l}\text { Interval, Site, } \\
\text { Core, Section, } \\
\text { Subbottom } \\
\text { Depth (m) }\end{array}$ & Events & \multirow{2}{*}{ 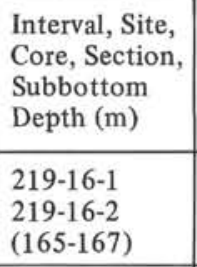 } & Events & $\begin{array}{l}\text { Interval, Site, } \\
\text { Core, Section, } \\
\text { Subbottom } \\
\text { Depth (m) }\end{array}$ \\
\hline & & & $\begin{array}{l}\text { B Globigerina } \\
\text { angustiumbilicata }\end{array}$ & & & \\
\hline & & & B Tenuitella gemma & $\begin{array}{l}219-16-1 \\
219-16-2 \\
(165-167)\end{array}$ & & \\
\hline & & & $\begin{array}{l}\mathrm{T} \text { Hantkenina (Hantkenina) } \\
\text { alabamensis }\end{array}$ & $\begin{array}{l}219-16-2 \\
219-16-3 \\
(167-168)\end{array}$ & & \\
\hline & & & $\begin{array}{l}\text { B Globoquadrina tripartita } \\
\text { tapuriensis }\end{array}$ & $\begin{array}{l}219-16-3 \\
219-16-4 \\
(168-170)\end{array}$ & & \\
\hline & & & T Pseudohastigerina micra & $\begin{array}{l}219-16-3 \\
219-16-4 \\
(168-170)\end{array}$ & & \\
\hline & & & $\begin{array}{l}\text { T Turborotalia (Turborotalia) } \\
\text { cerroazulensis } \\
\text { cerroazulensis }\end{array}$ & $\begin{array}{l}219-16-3 \\
219-16-4 \\
(168-170)\end{array}$ & & \\
\hline & & & $\begin{array}{l}\text { T Turborotalia (Turborotalia) } \\
\text { cerroazulensis cocoaensis }\end{array}$ & $\begin{array}{l}219-16-3 \\
219-16-4 \\
(168-170)\end{array}$ & & \\
\hline & & & $\begin{array}{l}\text { Turborotalia (Turborotalia) } \\
\text { cerroazulensis pomeroli }\end{array}$ & $\begin{array}{l}219-16-3 \\
219-16-4 \\
(168-170)\end{array}$ & & \\
\hline & & & B Subbotina winkleri & $\begin{array}{l}219-16-4 \\
219-16-5 \\
(170-171)\end{array}$ & & \\
\hline & & & $\begin{array}{l}\text { B Turborotalia (Turborotalia) } \\
\text { prasaepis }\end{array}$ & $\begin{array}{l}219-16-6 \\
219-16, \mathrm{CC} \\
(173-174)\end{array}$ & & \\
\hline & & & $\begin{array}{l}\text { B Turborotalia (Turborotalia) } \\
\text { pseudoampliapertura }\end{array}$ & $\begin{array}{l}219-16-6 \\
219-16, \mathrm{CC} \\
(173-174)\end{array}$ & & \\
\hline \multirow[t]{8}{*}{$\begin{array}{l}\text { T Discoaster } \\
\text { barbadiensis }\end{array}$} & \begin{tabular}{l|}
$220-10, \mathrm{CC}$ \\
$220-11-1$ \\
$(162-198)$ \\
\end{tabular} & $\begin{array}{l}219-16, \mathrm{CC} \\
219-17-1 \\
(173-174) \\
\end{array}$ & $\begin{array}{l}\text { B Turborotalia (Turborotalia) } \\
\text { opima nana }\end{array}$ & $\begin{array}{l}219-16, \mathrm{CC} \\
219-17-1 \\
(173-174) \\
\end{array}$ & $\begin{array}{l}\text { B Dorcadospyris } \\
\text { spinosa }\end{array}$ & $\begin{array}{l}220-10, \mathrm{CC} \\
220-11-1 \\
(162-198) \\
\end{array}$ \\
\hline & & & T Subbotina linaperta & $\begin{array}{l}219-16, \mathrm{CC} \\
219-17-1 \\
(173-174)\end{array}$ & $\begin{array}{l}\text { B Theocorys } \\
\text { spongoconum }\end{array}$ & $\begin{array}{l}220-10, \mathrm{CC} \\
220-11-1 \\
(162-198)\end{array}$ \\
\hline & & & & & $\begin{array}{l}\mathrm{T} \text { Lychnocanoma } \\
\text { babylonis group }\end{array}$ & $\begin{array}{l}220-10, \mathrm{CC} \\
220-11-1 \\
(162-198)\end{array}$ \\
\hline & & & & & B Theocampe pirum & $\begin{array}{l}220-10, \mathrm{CC} \\
220-11-1 \\
(162-198)\end{array}$ \\
\hline & & & & & $\begin{array}{l}\mathrm{T} \text { Theocampe } \\
\text { mongolfieri }\end{array}$ & $\begin{array}{l}220-10, \mathrm{CC} \\
220-11-1 \\
(162-198)\end{array}$ \\
\hline & & & & & $\begin{array}{l}\text { T Lophocyrtis (?) } \\
\text { jacchia }\end{array}$ & $\begin{array}{l}220-10, C C \\
220-11-1 \\
(162-198)\end{array}$ \\
\hline & & & & & $\begin{array}{l}\mathrm{T} \text { Theocampe } \\
\text { armadillo }\end{array}$ & $\begin{array}{l}220-10, \mathrm{CC} \\
220-11-1 \\
(162-198) \\
\end{array}$ \\
\hline & & & & & $\begin{array}{l}\text { T Periphaena } \\
\text { decora }\end{array}$ & $\begin{array}{l}220-10, \mathrm{CC} \\
220-11-1 \\
(162-198)\end{array}$ \\
\hline
\end{tabular}


TABLE 1 - Continued

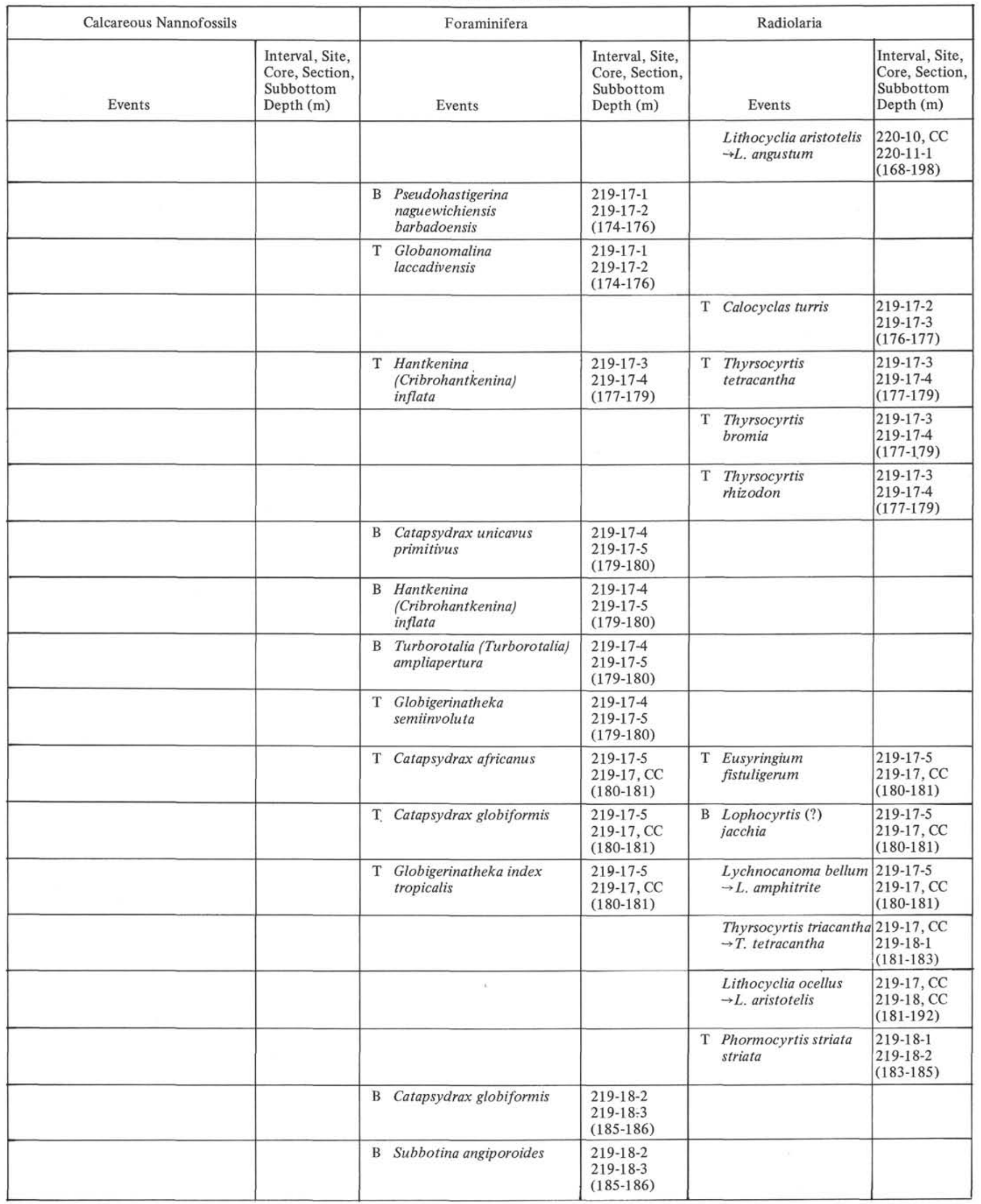


TABLE 1 - Continued

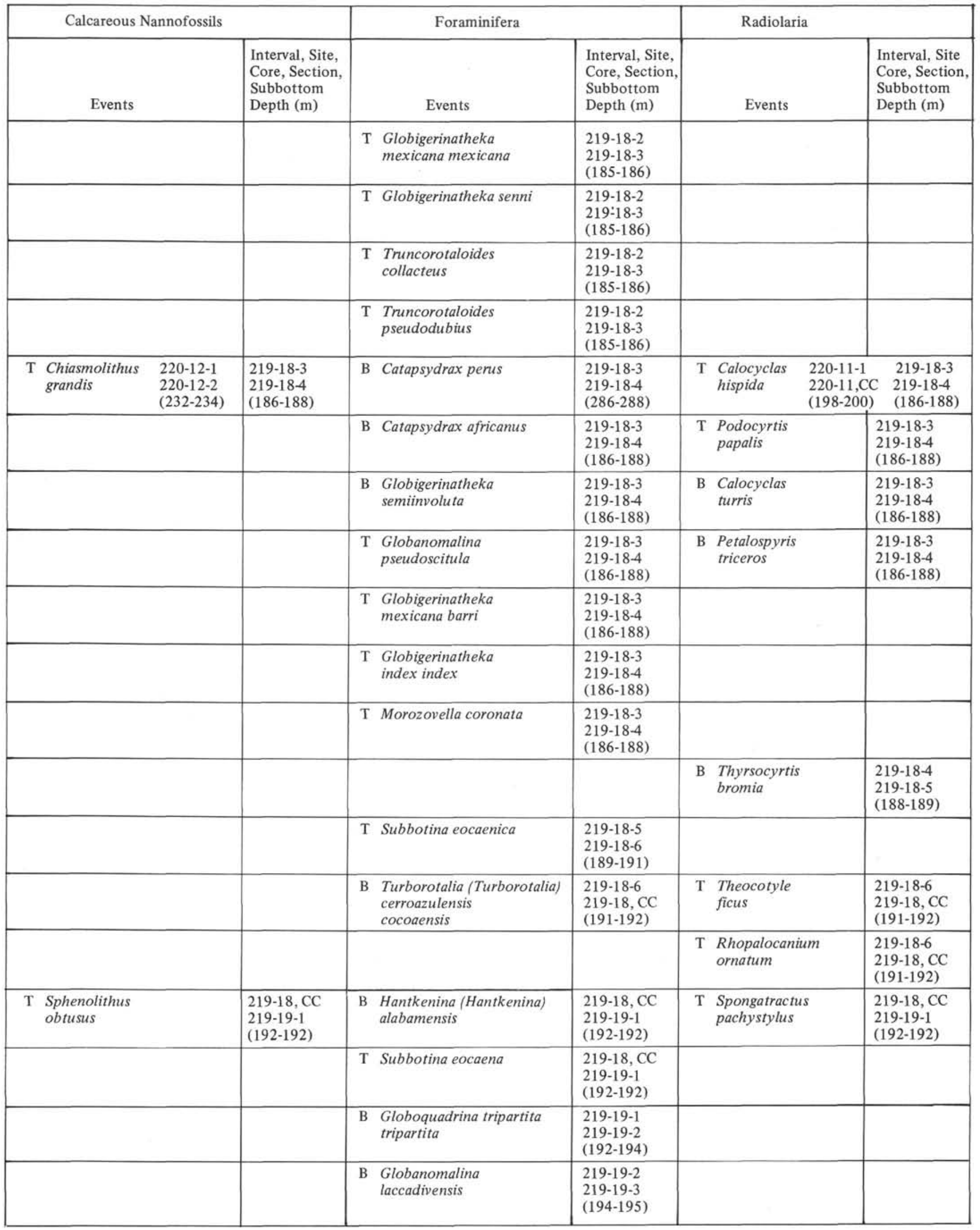


TABLE 1 - Continued

\begin{tabular}{|c|c|c|c|c|c|}
\hline \multicolumn{2}{|c|}{ Calcareous Nannofossil } & \multicolumn{2}{|l|}{ Foraminifera } & \multicolumn{2}{|l|}{ Radiolaria } \\
\hline Events & $\begin{array}{l}\text { Interval, Site, } \\
\text { Core, Section, } \\
\text { Subbottom } \\
\text { Depth (m) }\end{array}$ & Events & $\begin{array}{l}\text { Interval, Site, } \\
\text { Core, Section, } \\
\text { Subbottom } \\
\text { Depth (m) }\end{array}$ & Events & $\begin{array}{l}\text { Interval, Site } \\
\text { Core, Section, } \\
\text { Subbottom } \\
\text { Depth (m) }\end{array}$ \\
\hline & & $\begin{array}{l}\text { B Globigerinatheka index } \\
\text { tropicalis }\end{array}$ & $\begin{array}{l}219-19-3 \\
219-19-4 \\
(195-197)\end{array}$ & & \\
\hline & & $\begin{array}{l}\text { B Turborotalia (Turborotalia) } \\
\text { cerroazulensis } \\
\text { cerroazulensis }\end{array}$ & $\begin{array}{l}219-19-3 \\
219-19-4 \\
(195-197)\end{array}$ & & \\
\hline & & & & $\begin{array}{l}\text { T Lithochytris } \\
\text { vespertilio }\end{array}$ & $\begin{array}{l}219-19-4 \\
219-19-5 \\
(197-198)\end{array}$ \\
\hline & & B Globoquadrina galavisi & $\begin{array}{l}219-19-5 \\
219-19-6 \\
(198-200)\end{array}$ & T Heliostylus spp. & $\begin{array}{l}219-19-5 \\
219-19, \text { CC } \\
(198-201)\end{array}$ \\
\hline & & B Globorotaloides suteri & $\begin{array}{l}219-19-5 \\
219-19-6 \\
(198-200)\end{array}$ & B Podocyrtis mitra & $\begin{array}{l}219-19-5 \\
219-19, \mathrm{CC} \\
(198-201)\end{array}$ \\
\hline & & B Subbotina linaperta & $\begin{array}{l}219-19-5 \\
219-19-6 \\
(198-200)\end{array}$ & $\begin{array}{l}\text { T Phormocyrtis } \\
\text { embolum }\end{array}$ & $\begin{array}{l}219-19-5 \\
219-19, \mathrm{CC} \\
(198-201)\end{array}$ \\
\hline & & T Acarinina boudreauxi & $\begin{array}{l}219-19-5 \\
219-19-6 \\
(198-200)\end{array}$ & $\begin{array}{l}\mathrm{T} \text { Podocyrtis } \\
\text { trachodes }\end{array}$ & $\begin{array}{l}219-19-5 \\
219-19, \mathrm{CC} \\
(198-201)\end{array}$ \\
\hline & & $\begin{array}{l}\text { T Globigerinatheka } \\
\text { subconglobata curryi }\end{array}$ & $\begin{array}{l}219-19-5 \\
219-19-6 \\
(198-200)\end{array}$ & $=$ & \\
\hline & & T Morozovella bandyi & $\begin{array}{l}219-19-5 \\
219-19-6 \\
(198-200)\end{array}$ & & \\
\hline & & T Subbotina kiersteadae & $\begin{array}{l}219-19-5 \\
219-19-6 \\
(198-200)\end{array}$ & & \\
\hline & & $\mathrm{T}$ Acarinina planodorsalis & $\begin{array}{l}219-19-6 \\
219-19, \mathrm{CC} \\
(200-201)\end{array}$ & & \\
\hline & & $\mathrm{T}$ Acarinina punctocarinata & $\begin{array}{l}219-19-6 \\
219-19, \mathrm{CC} \\
(200-201)\end{array}$ & & \\
\hline & & $\begin{array}{l}\mathrm{T} \text { Morozovella aragonensis } \\
\text { aragonensis }\end{array}$ & $\begin{array}{l}219-19-6 \\
219-19, \mathrm{CC} \\
(200-201)\end{array}$ & & \\
\hline & & $\begin{array}{l}\text { T Pseudohastigerina } \\
\text { wilcoxensis }\end{array}$ & $\begin{array}{l}219-19-6 \\
219-19, \mathrm{CC} \\
(200-201)\end{array}$ & & \\
\hline & & T Subbotina patagonica & $\begin{array}{l}219-19-6 \\
219-19, \mathrm{CC} \\
(200-201)\end{array}$ & & \\
\hline & & B Pseudohastigerina micra & $\begin{array}{l}219-19, \mathrm{CC} \\
219-20-1 \\
(201-201) \\
\end{array}$ & $\begin{array}{l}\text { B Theocampe } \\
\text { armadillo }\end{array}$ & $\begin{array}{l}219-19, \mathrm{CC} \\
219-20-1 \\
(201-201) \\
\end{array}$ \\
\hline & & & & $\begin{array}{l}\mathrm{T} \text { Podocyrtis } \\
\text { ampla fasciolata }\end{array}$ & $\begin{array}{l}219-19, \mathrm{CC} \\
219-20-1 \\
(201-201) \\
\end{array}$ \\
\hline & & $\mathrm{T}$ Acarinina bulbrooki & $\begin{array}{l}219-20-1 \\
219-20-2 \\
(201-203)\end{array}$ & & \\
\hline & & $\begin{array}{l}\text { B Globigerinatheka } \\
\text { subconglobata curryi }\end{array}$ & $\begin{array}{l}219-20-2 \\
219-20-3 \\
(203-204)\end{array}$ & $\begin{array}{l}\mathrm{T} \text { Lophocyrtis } \\
\text { biaurita }\end{array}$ & $\begin{array}{l}219-20-2 \\
219-20-3 \\
(203-204)\end{array}$ \\
\hline
\end{tabular}


C. NIGRINI, R. FLEISHER, J. BOUDREAUX

TABLE 1 - Continued

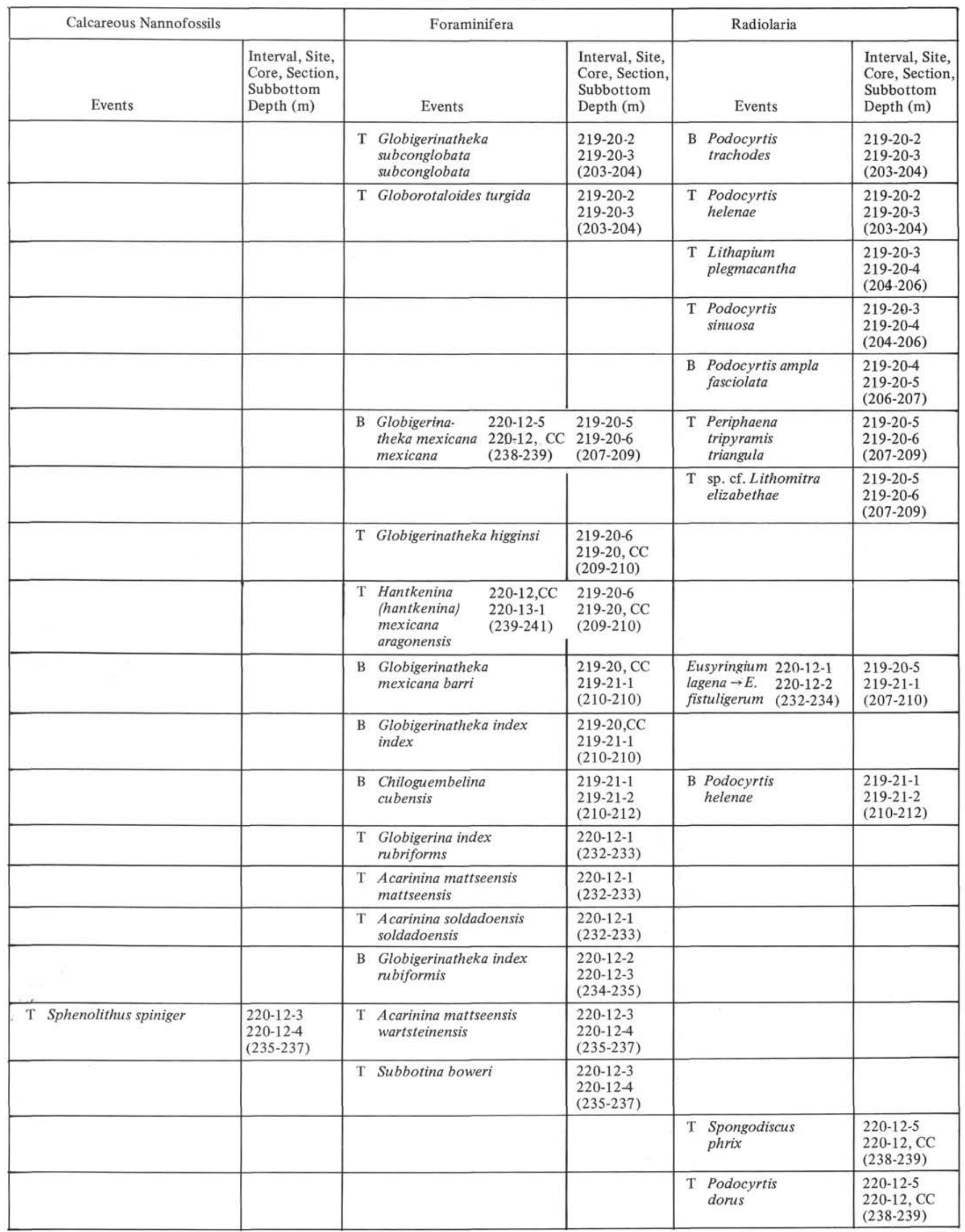


TABLE 1 - Continued

\begin{tabular}{|c|c|c|c|c|c|}
\hline \multicolumn{2}{|c|}{ Calcareous Nannofossils } & \multicolumn{2}{|l|}{ Foraminifera } & \multicolumn{2}{|l|}{ Radiolaria } \\
\hline \multirow[t]{6}{*}{ Events } & $\begin{array}{l}\text { Interval, Site, } \\
\text { Core, Section, } \\
\text { Subbottom } \\
\text { Depth (m) }\end{array}$ & Events & $\begin{array}{l}\text { Interval, Site, } \\
\text { Core, Section, } \\
\text { Subbottom } \\
\text { Depth }(\mathrm{m})\end{array}$ & Events & $\begin{array}{l}\text { Interval, Site, } \\
\text { Core, Section, } \\
\text { Subbottom } \\
\text { Depth (m) }\end{array}$ \\
\hline & & & & $\begin{array}{l}\text { T Theocotyle } \\
\text { cryptocephala } \\
\text { conica }\end{array}$ & $\begin{array}{l}220-12, \mathrm{CC} \\
220-13-1 \\
(239-241)\end{array}$ \\
\hline & & B Morozovella coronata & $\begin{array}{l}220-13-2 \\
220-13-3 \\
(243-244)\end{array}$ & & \\
\hline & & $\begin{array}{l}\text { B Hantkenina (hantkenina) } \\
\text { mexicana aragonensis }\end{array}$ & $\begin{array}{l}220-13-3 \\
220-13-4 \\
(244-246)\end{array}$ & & \\
\hline & & B Morozovella bandyi & $\begin{array}{l}220-13-3 \\
220-13-4 \\
(244-246)\end{array}$ & & \\
\hline & & $\begin{array}{c}\text { B Globigerinatheka } \\
\text { subconglobata } \\
\text { subconglobata }\end{array}$ & $\begin{array}{l}220-13-4 \\
220-13, \mathrm{CC} \\
(246-247)\end{array}$ & & \\
\hline \multirow[t]{12}{*}{$\begin{array}{l}\text { T Chiasmolithus } \\
\text { gigas }\end{array}$} & $\begin{array}{l}220-13, \mathrm{CC} \\
220-14-1 \\
(247-250)\end{array}$ & B Acarinina planodorsalis & $\begin{array}{l}220-13, \mathrm{CC} \\
220-14-1 \\
(247-250)\end{array}$ & $\begin{array}{l}\text { B Eusyringium } \\
\text { lagena }\end{array}$ & $\begin{array}{l}220-13, \mathrm{CC} \\
220-14-1 \\
(247-250) \\
\end{array}$ \\
\hline & & $\begin{array}{l}\text { B Globigerinatheka index } \\
\text { index }\end{array}$ & $\begin{array}{l}220-13, \mathrm{CC} \\
220-14-1 \\
(247-250)\end{array}$ & $\begin{array}{l}\mathrm{T} \text { Amphicraspedum } \\
\text { prolixum }\end{array}$ & $\begin{array}{l}220-13, \mathrm{CC} \\
220-14-1 \\
(247-250)\end{array}$ \\
\hline & & $\begin{array}{l}\text { B Turborotalia (turborotalia) } \\
\text { cerroazulensis pomeroli }\end{array}$ & $\begin{array}{l}220-13, \mathrm{CC} \\
220-14-1 \\
(247-250)\end{array}$ & $\begin{array}{l}\text { B Podocyrtis } \\
\text { dorus }\end{array}$ & $\begin{array}{l}220-13, \mathrm{CC} \\
220-14-1 \\
(247-250) \\
\end{array}$ \\
\hline & & & & $\begin{array}{l}\text { Theocotyle crypto- } \\
\text { cephala } \rightarrow T . \text { c. conica }\end{array}$ & $\begin{array}{l}220-13, \mathrm{CC} \\
220-14-3 \\
(247-253)\end{array}$ \\
\hline & & $\begin{array}{l}\text { B Truncorotaloides } \\
\text { pseudodubius }\end{array}$ & $\begin{array}{l}220-14-1 \\
220-14-2 \\
(250-252)\end{array}$ & $\begin{array}{l}\text { Thyrsocyrtis hirsuta } \\
\text { tensa } \rightarrow T \text {. triacantha }\end{array}$ & $\begin{array}{l}220-14-1 \\
220-14, \mathrm{CC} \\
(250-255)\end{array}$ \\
\hline & & & & $\begin{array}{l}\text { T Lamptonium } \\
\text { fabaeforme (?) } \\
\text { chaunothorax }\end{array}$ & $\begin{array}{l}220-14-2 \\
220-14-3 \\
(252-253) \\
\end{array}$ \\
\hline & & B Subbotina eocaenica & $\begin{array}{l}220-14-3 \\
220-14, \mathrm{CC} \\
(253-255)\end{array}$ & $\begin{array}{l}\text { Thyrsocyrtis } \\
\text { hirsuta hirsuta }\end{array}$ & $\begin{array}{l}220-14-3 \\
220-14, \mathrm{CC} \\
(253-255)\end{array}$ \\
\hline & 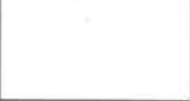 & $\begin{array}{l}\text { B Truncorotaloides } \\
\text { collacteus }\end{array}$ & $\begin{array}{l}220-14-3 \\
220-14 ; \mathrm{CC} \\
(253-255)\end{array}$ & $\begin{array}{l}\text { T Lamptonium } \\
\text { fabaeforme }(?) \\
\text { constrictum }\end{array}$ & $\begin{array}{l}220-14-3 \\
220-14, \mathrm{CC} \\
(253-255)\end{array}$ \\
\hline & & & . & T Periphaena delta & $\begin{array}{l}220-14-3 \\
220-14, \mathrm{CC} \\
(253-255)\end{array}$ \\
\hline & & & & $\begin{array}{l}\mathrm{T} \text { Thyrsocyrtis } \\
\text { hirsuta robusta }\end{array}$ & $\begin{array}{l}220-14-3 \\
220-14, \mathrm{CC} \\
(253-255) \\
\end{array}$ \\
\hline & & $\mathrm{T}$ Acarinina pentacamerata & $\begin{array}{l}220-14, \mathrm{CC} \\
220-15-2 \\
(255-261)\end{array}$ & $\begin{array}{l}\text { T Stylotrochus } \\
\text { quadribrachiatus } \\
\text { quadribrachiatus }\end{array}$ & $\begin{array}{l}220-14, \mathrm{CC} \\
220-15-2 \\
(255-261) \\
\end{array}$ \\
\hline & . & $\begin{array}{l}\text { T Acarinina soldadoensis } \\
\text { angulosa }\end{array}$ & $\begin{array}{l}220-15-2 \\
220-15-3 \\
(261-262)\end{array}$ & $\begin{array}{l}\mathrm{T} \text { Stylosphaera } \\
\text { coronata sabaca }\end{array}$ & $\begin{array}{l}220-15-2 \\
220-15-3 \\
(261-262) \\
\end{array}$ \\
\hline \multirow[t]{2}{*}{$\begin{array}{l}\text { T Discoaster } \\
\text { sublodoensis }\end{array}$} & $\begin{array}{l}220-14-3 \\
220-15-4 \\
(262-264) \\
\end{array}$ & B Acarinina punctocarinata & $\begin{array}{l}220-15-3 \\
220-15-4 \\
(262-264)\end{array}$ & $\begin{array}{l}\mathrm{T} \text { Lamptontium } \\
\text { fabaeforme } \\
\text { fabaeforme (?) }\end{array}$ & $\begin{array}{l}220-15-3 \\
220-15-4 \\
(262-264)\end{array}$ \\
\hline & & & & $\begin{array}{l}\text { B Lithapium } \\
\text { plegmacantha }\end{array}$ & $\begin{array}{l}220-15-3 \\
220-15-4 \\
(262-264)\end{array}$ \\
\hline
\end{tabular}


TABLE 1 - Continued

\begin{tabular}{|c|c|c|c|c|c|}
\hline \multicolumn{2}{|c|}{ Calcareous Nannofossils } & \multicolumn{2}{|l|}{ Foraminifera } & \multicolumn{2}{|l|}{ Radiolaria } \\
\hline \multirow[t]{6}{*}{ Events } & $\begin{array}{l}\text { Interval, Site, } \\
\text { Core, Section, } \\
\text { Subbottom } \\
\text { Depth (m) }\end{array}$ & Events & $\begin{array}{l}\text { Interval: Site } \\
\text { Core, Section, } \\
\text { Subbottom } \\
\text { Depth }(\mathrm{m})\end{array}$ & Events & $\begin{array}{l}\text { Interval: Site, } \\
\text { Core, Section, } \\
\text { Subbottom } \\
\text { Depth (m) }\end{array}$ \\
\hline & & $\begin{array}{l}\text { B Acarinina mattseensis } \\
\text { wartsteinensis }\end{array}$ & \begin{tabular}{|l|}
$220-15-4$ \\
$220-15-5$ \\
$(264-265)$
\end{tabular} & & \\
\hline & & $\mathrm{T}$ Acarinina apanthesma & \begin{tabular}{|l|}
$220-15-5$ \\
$220-15-6$ \\
$(265-267)$
\end{tabular} & & \\
\hline & & & & $\begin{array}{l}\text { Lithochytris archaea } \\
\rightarrow \text { L. vespertilio }\end{array}$ & $\begin{array}{l}220-15-5 \\
220-16-2 \\
(265-290)\end{array}$ \\
\hline & & & & $\begin{array}{l}\text { Theocotyle crypto- } \\
\text { cephala nigriniae } \\
\rightarrow T . \text { c. cryptocephala }\end{array}$ & $\begin{array}{l}220-15-5 \\
220-16-2 \\
(265-290)\end{array}$ \\
\hline & & & & $\begin{array}{l}\text { B Stylotrochus } \\
\text { quadribrachiatus } \\
\text { quadribrachiatus } \\
\end{array}$ & $\begin{array}{l}220-15-6 \\
220-15, \mathrm{CC} \\
(266-267) \\
\end{array}$ \\
\hline \multirow[t]{11}{*}{$\mathrm{T}$ Discoaster lodoensis } & $\begin{array}{l}220-15, \mathrm{CC} \\
220-16-2 \\
(267-290)\end{array}$ & B Acarinina boudreauxi & $\begin{array}{l}220-15, \mathrm{CC} \\
220-16-2 \\
(267-290)\end{array}$ & $\begin{array}{l}\text { B Theocampe } \\
\text { mongolfieri }\end{array}$ & $\begin{array}{l}220-15, \mathrm{CC} \\
220-16-2 \\
(267-290)\end{array}$ \\
\hline & & B Subbotina patagonica & $\begin{array}{l}220-15, C C \\
220-16-2 \\
(267-290)\end{array}$ & & \\
\hline & & B Subbotina boweri & $\begin{array}{l}220-15, C C \\
220-16-2 \\
(267-290)\end{array}$ & & \\
\hline & & $\begin{array}{l}\mathrm{T} \text { Acarinina mattseensis } \\
\text { alticonica }\end{array}$ & $\begin{array}{l}220-15, \mathrm{CC} \\
220-16-2 \\
(267-290)\end{array}$ & & \\
\hline & & $\begin{array}{l}\mathrm{T} \text { Morozovella aragonensis } \\
\text { caucasica }\end{array}$ & $\begin{array}{l}220-15, \mathrm{CC} \\
220-16-2 \\
(267-290)\end{array}$ & & \\
\hline & & B Globigerinatheka senni & \begin{tabular}{|l}
$220-16-2$ \\
$220-16, \mathrm{CC}$ \\
$(267-290)$
\end{tabular} & $\mathrm{T}$ Buryella clinata & $\begin{array}{l}220-16-2 \\
220-16, \mathrm{CC} \\
(290-290) \\
\end{array}$ \\
\hline & & $\mathrm{T}$ Acarinina wilcoxensis & \begin{tabular}{|l|}
$220-16-2$ \\
$220-16, C C$ \\
$(267-290)$
\end{tabular} & & \\
\hline & & & & $\begin{array}{l}\text { T Spongodiscus sp. } \\
\text { aff. S. cruciferus }\end{array}$ & $\begin{array}{l}220-16, C C \\
220-17-1 \\
(290-297) \\
\end{array}$ \\
\hline & & & & $\begin{array}{l}\mathrm{T} \text { Amphicraspedum } \\
\text { murrayanum }\end{array}$ & $\begin{array}{l}220-16, C C \\
220-17-1 \\
(290-297) \\
\end{array}$ \\
\hline & & $\begin{array}{l}\text { B Acarinina mattseensis } \\
\text { mattseensis }\end{array}$ & $\begin{array}{l}220-17-1 \\
220-18-2 \\
(297-328)\end{array}$ & & \\
\hline & & $\mathrm{T}$ Acarinina convexa & \begin{tabular}{|l|}
$220-17-1$ \\
$220-18-2$ \\
$(297-328)$ \\
\end{tabular} & & \\
\hline \multirow[t]{3}{*}{$\begin{array}{l}\text { T Marthasterites } \\
\text { tribrachiatus }\end{array}$} & \begin{tabular}{|l}
$220-17, \mathrm{CC}$ \\
$220-18-2$ \\
$(299-328)$ \\
\end{tabular} & & & & \\
\hline & & B Globorotaloides turgida & \begin{tabular}{|l|}
$220-18-2$ \\
$220-18-3$ \\
$(328-329)$ \\
\end{tabular} & & \\
\hline & & $\mathrm{T}$ Acarinina quetra & $\begin{array}{l}220-18-2 \\
220-18-3 \\
(328-329)\end{array}$ & & \\
\hline
\end{tabular}


TABLE 2

Relationship of Microfossil Zones Observed at Sites 219 and 220

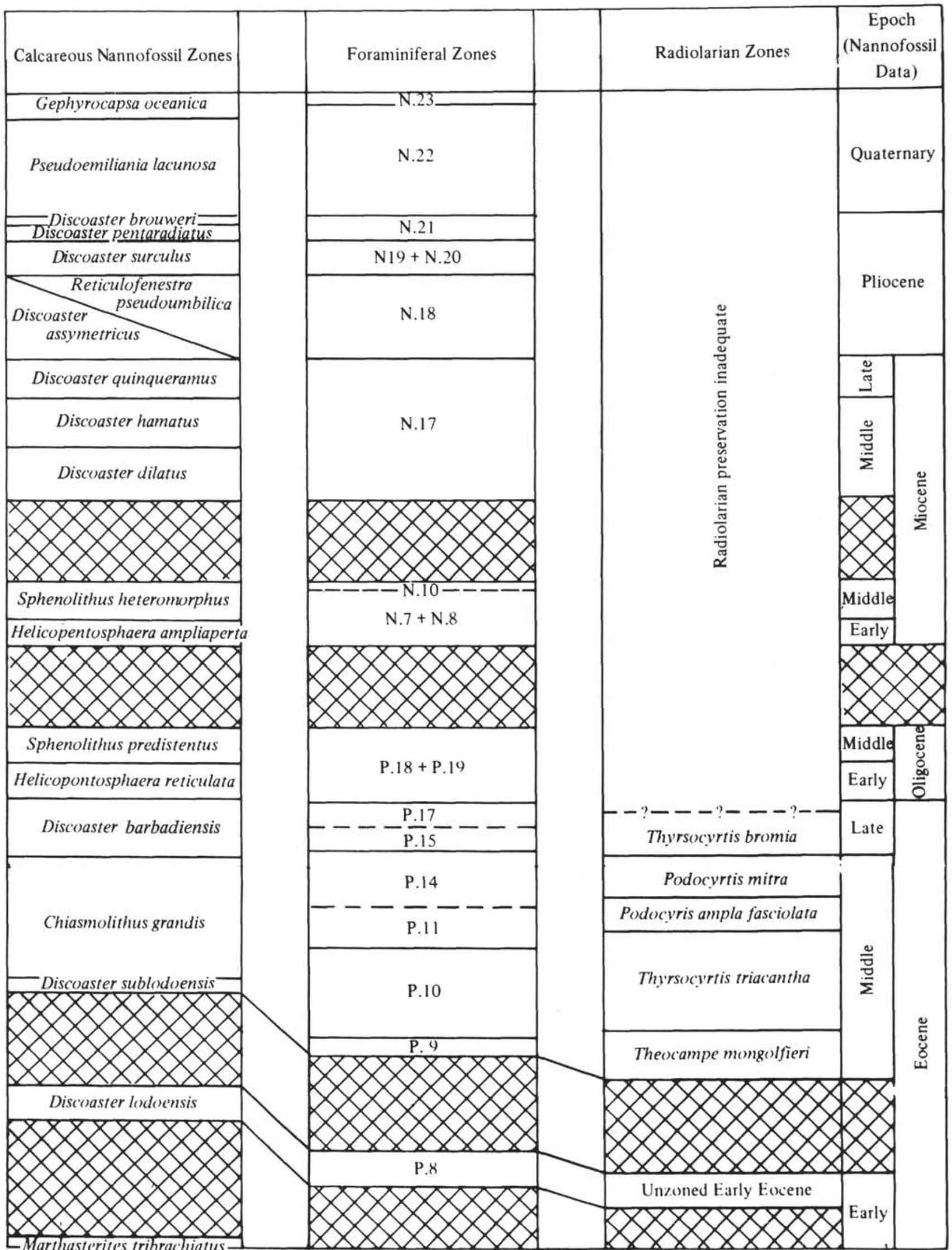

Note: See text for a detailed explanation of table. 